\title{
THE DISTRIBUTION OF M SUPERGIANTS WITH LARGE INFRARED EXCESS AND WR STARS IN THE GALAXY
}

\author{
M. Raharto \\ Bosscha Observatory and Department of Astronomy \\ Bandung Institute of Technology \\ Indonesia
}

Summary. From the $m_{12}-m_{25}$ vs. spectral type diagram (see for example: Habing, 1987, Figure 5), it can be seen that there are no early $M$ giant stars with $m_{12}-m_{25}>$ 0.7 . On the other hand all early $M$ stars with $m_{12}-m_{25}>0.7$ are $M$ supergiants. These early type $M$ supergiants have typically infrared excesses at $12 \mu \mathrm{m}$ in the range of 20 to 170 times higher than their photosperic fluxes.

The color distinction between early $M$ giants and $M$ supergiants was applied to identify early $M$ giant stars from the Catalogue of Spectral Classifications for Stars of the Catalogue of the Caltech-Two-Micron Survey by Bidelman (1980). The search was confined to $l=345^{\circ}-300^{\circ}$, through the galactic centre direction. Only good data were used, i.e. for objects with flux quality 3 in the scale of IRAS (1984). There are 182 stars classified as $M$ supergiants according to the above mentioned criteria, 58 of which are known spectroscopically as $M$ supergiants. The surface distribution of the $M$ supergiants found is compared to the surface distribution of Wolf Rayet (WR) stars listed in van der Hucht et al. (1988). The distribution shows four clumpings of $M$ supergiants, at $\left(0^{\circ}<l<\right.$ $\left.30^{\circ},-2^{\circ}<b<+2^{\circ}\right),\left(60^{\circ}<l<90^{\circ},-1^{\circ}<b<+3^{\circ}\right),\left(100^{\circ}<l<120^{\circ},-2^{\circ}<b<+2^{\circ}\right)$ and $\left(120^{\circ}<l<150^{\circ},-5^{\circ}<b<+0^{\circ}\right)$. The first three are found in the direction of high concentrations of WR stars.

In order to know whether there is a spatial coincidence between these two types of stars, the distance estimates of $M$ supergiants were calculated. The distance estimation was facilitated by the calibration of the absolute magnitude of $\mathrm{M}$ supergiants at $12 \mu \mathrm{m}$ which was assumed to be a liniar function of $m_{12}-m_{25}$ (Raharto, 1990) and the interstellar extinction at $12 \mu \mathrm{m}$ was negligible.

Then the distributions of the two types of stars within $3 \mathrm{kpc}$ from the Sun were compared. Two apparent clumpings of $M$ supergiants coincide with the WR space distribution given by van der Hucht et al. (1988). The clumpings of $M$ supergiants at $\left(100^{\circ}<l<120^{\circ},-2^{\circ}<\right.$ $\left.b<+2^{\circ}\right)$ and $\left(120^{\circ}<l<150^{\circ},-5^{\circ}<b<+0^{\circ}\right)$, however, do not coincide with any of the WR concentrations. It has been known that this direction is devoid of WR stars (Roberts, 1962). The clumping of $M$ supergiants in this direction may indicate that the IMF in different spiral arms of the Galaxy may not the same.

\section{Acknowledgements}

The author would like to thank the LOC of the IAU Symposium no. 143 for providing financial support to attend the meeting as well as their hospitality during the meeting. The

K. A. van der Hucht and B. Hidayat (eds.),

Wolf-Rayet Stars and Interrelations with Other Massive Stars in Galaxies, 641-642.

(c) 1991 IAU. Printed in the Netherlands. 
author is also indebted to Prof. B. Hidayat for his critical comments and suggestions on the draft of the manuscript. The author would also like to express thanks to Prof. Habing, Dr. Piet Schwering and their colleagues in Leiden Sterrewacht for their hospitality and various help during a two year stay (1984-1986) in Leiden where this work was initiated. A part of this works was financially supported by Institute for Research - Bandung Institute of Technology.

\section{References}

Bidelman, W.P., 1980, Publications of the Warner and Swasey Observatory, Vol. 2.

Habing, H.J., 1987, Proc. IAU Symposium No. 122, Circumstellar Matter, I. Appenzeller and C. Jordan (eds.), D. Reidel Publ. Co., p. 197.

van der Hucht, K.A., Hidayat, B., Admiranto, G, Supelli, K.R., Doom, C., 1988 Astron. Astrophys. 199, 217.

IRAS, 1984, The Explanatory Suplement, eds. C.A. Beichman, G. Neugebauer, H.J. Habing, P.E. Clegg, T.J. Chester (Goverment Printing Office).

Raharto, M., 1990, in Proc. 5th IAU Asian Pacific Regional Meeting Sydney-Australia, 1990 , in press.

Roberts, M., 1962, Astron. J. 67, 79. 